\title{
Experimental study of the drying and modelling of the humidity migration in a clay matrix
}

\author{
K.-E. Atcholi ${ }^{1, a}$, E. Padayodi ${ }^{1}$, J. Vantomme ${ }^{2}$, K. Kadja ${ }^{1}$ and D. Perreux ${ }^{3}$ \\ 1 Laboratoire d'Études et de Recherches sur les Matériaux, les Procédés et les Surfaces (LERMPS) \\ Équipe de Recherche sur les Agro-Matériaux et la Santé Environnementale (ERAMSE), \\ Département de Génie Mécanique et Conception, Université de Technologie de Belfort-Montbéliard, \\ 90010 Belfort Cedex, France \\ 2 Civil Engineering Department, École Royale Militaire de Bruxelles, Belgique \\ 3 Laboratoire de Mécanique Appliquée, R. Chaléat (LMARC), Université de Franche-Comté, 25030 Besançon, France
}

Received 18 June 2007, accepted 2 January 2008

\begin{abstract}
Available almost everywhere, therefore economic and even so durable, clays have stimulated the creativity of men who have imagined since millennia and under all latitudes, multiple processes to develop pottery (faïence enameled, porcelain, ceramics) and construction materials. These materials compete with modern materials in civil engineering for important construction works such as bridges, roads, tunnels, monuments etc. In state-of-the-art technologies, clay ceramics are used as anti-thermal coatings: turbine vanes, etc... The weakness of our scientific knowledge limits applications of these natural materials. This study on tropical clays, has for objective on one hand to develop new processing of construction materials for lodging needs that are more and more increasing in the developing countries, and the other hand to develop new refractory materials, anti-thermal coatings or ceramic prostheses. This study is part of a research program and academic cooperation between the laboratories LMARC-Besançon/France, LERMPS-Belfort/France, Civil Eng. Dept./ERM-Bruxelles/Belgique, URMA-Lomé/Togo (K.-E. Atcholi, Rapport d'activités de Recherches, Université de Lomé, Février 1997). In this paper, we present an experimental modelling of a clay matrix drying in order to better understand and resolve material deformations and cracking. The complex behaviour of the clay during drying, leads us to propose two complementary approaches: an experimental approach that allows to establish the drying kinetic and a modelling of moisture transfer in a clay matrix in order to optimise the drying. A diffusive model based on Fick's laws allowed to highlight the internal stresses causing matrixes damage.
\end{abstract}

Key words: Clay; dough; drying; building materials; diffusion laws

\section{Introduction}

In the process of clay materials manufacture, drying is a critical operation that determines the resulting products quality. So, this operation requires particular cautions taking in account the complex micro and macro transformations occurring inside the clay material, and that generate material damaging such deformations and cracks.

The clay matrix moisture evaporation is correlatively related to the matrix dimensions variations by shrinking and to the matrix densification. These related phenomena are the principle causes of the material damage during drying.

Understanding the mechanisms of a clay matrix shrinking, densification and evaporation will make it pos-

\footnotetext{
a Corresponding author:

kokou-esso.atcholi@utbm.fr, katcholi@hotmail.com
}

sible to overcome to these damages or to reduce damage risks during drying.

Due to the complexity of these phenomena occurring in a clay matrix during drying, we propose in this study two complementary approaches: an experimental approach that allowed the quantification of the phenomena at the macroscopic scale and a theoretical approach that is about the modelling of the moisture transfer in a clay flat matrix during drying using a diffusive model.

\section{Experimental approach of a clay matrix drying}

This experimental approach deals with the matrix water content variation during drying and the matrix sizes variation due to the shrinkage. 


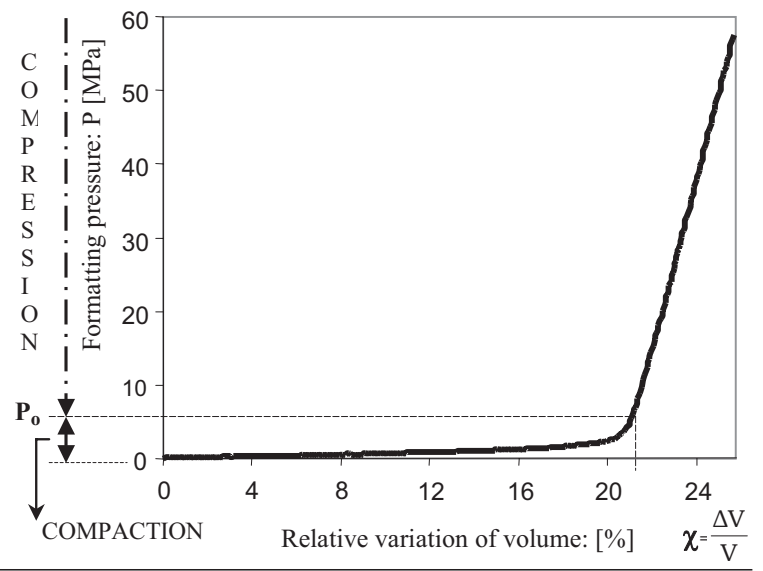

Fig. 1. Compaction curve of the clay paste: variation of the relative volume of the matrix in function of pressure.

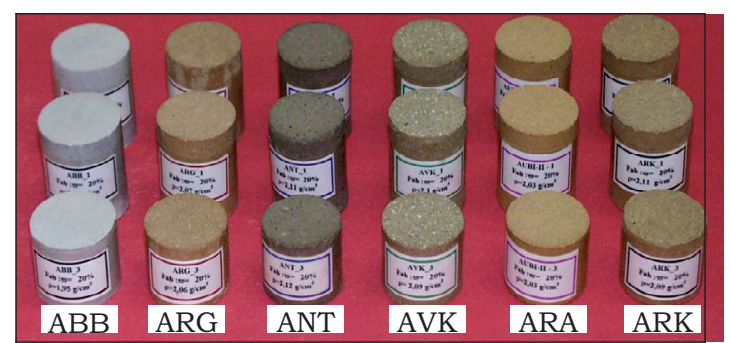

Fig. 2. Cylindrical test-tubes made of clay matrix. Six clay varieties: white clay of Bangéli (ABB); red clay of GuérinKouka (ARG); black clay of Togblékopé (ANT); green clay of Kouvé (AVK); red clay of Albi (ARA) and red clay of Kouvé (ARK).

\subsection{Elaboration of clay matrix test-tubes}

A sample of clay is dried, grinded and sieved by means of a sieve of $0.5 \mathrm{~mm}$ of aperture size. Clay powders are moistened to a suitable shaping water content (Padayodi [2]) of $18 \%$. The obtained pastes are hermetically sealed and kept in a wet place during 8 weeks.

The cylindrical test-tubes are shaped by static pressing on a conventional machine of traction and compression. They are compressed to present a wet matrix density of about $2 \mathrm{~g} / \mathrm{cm}^{3}$ (Collard [3], Ho-Yick-Cheong [4]), a density advised in brickyard and tilery.

Figure 1 shows the test-tubes compaction curve giving the volume variation according to the pressure. This curve shows that the matrix undergoes a maximum compaction for a pressure of $8 \mathrm{MPa}$ (Ho-Yick-Cheong [4]). At this pressure, the clay test-tubes or matrixes present a density of about $1.94 \mathrm{~g} / \mathrm{cm}^{3}$ to $2.1 \mathrm{~g} / \mathrm{cm}^{3}$ according to the clay variety. These values meet the "Industrial Development Center" requirements (ACP-CEE [5]). This center advise a density between $1.87 \mathrm{~g} / \mathrm{cm}^{3}$ and $2.2 \mathrm{~g} / \mathrm{cm}^{3}$.

Figure 2 is an illustration of test-tubes of six clay varieties elaborated according to the above protocol.

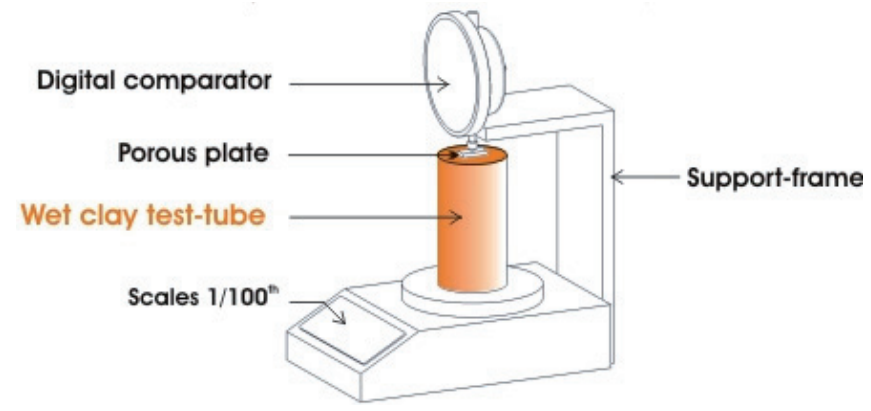

Fig. 3. Device for measuring the test-tube shrinkage and water content variation during drying.

\subsection{Clay matrixes drying: water content variation}

\subsubsection{Experimental devices and protocol}

The experimental device (Fig. 3) consists of an accurate scales on which lies the test-tube during drying and of a comparator that measures the test-tube shrinkage. A computer records the test-tube moisture evaporation according to the time and the test-tube size variation due to the shrinkage. The drying temperature is maintained between $20{ }^{\circ} \mathrm{C}$ and $22{ }^{\circ} \mathrm{C}$ and the relative moisture of the ambient air is maintained at $80 \%$.

\subsubsection{Results and discussions}

From the measured mass $m(t)$, one establishes for every clay the variation of the average water content $\varpi(t)$ of the matrix during drying. $\varpi(t)$ is given by the expression (Ho-Yick-Cheong [4], Magnan [6], Puffeney [7], Suri [8]):

$$
\varpi(t)=\frac{m(t)-m_{0}}{m_{0}}
$$

where $m_{o}$ is the dried sample mass. The derivative $d \varpi / d t$ gives is the drying velocity.

Figure 4 gives the curves of the average water content variation of different clay matrixes.

Table 1 gives the drying velocity at the beginning of drying and the balance water content $\varpi_{e q}$ at the end of drying. The balance water content $\varpi_{e q}$ is the residual water content of the matrix at the end of drying.

The experimental curves show that the clay matrix drying is carried out in two stages:

- A linear evaporation at the drying beginning. The moisture evaporation is intensive in this stage. The average values of the maximum drying velocity lie between -10 and $-5 \mathrm{mg} / \mathrm{h} . \mathrm{g}$ according to the clay variety. The negative sign of the drying velocity expresses the loss of moisture by evaporation.

According to Figure 4 and Table 1, the drying velocity is more important in the case of the ANT clay and lower for the ARG clay. This variation is related to their affinity with the moisture and the matrix porosity. 
Table 1. Drying velocity at the beginning of drying and the balance water content at the end of drying.

\begin{tabular}{ccccccc}
\hline & ABB & ANT & ARG & AVK & ARK & ARA \\
\hline $\begin{array}{c}\text { Drying velocity (mg /g of } \\
\text { dried matter / hour) }\end{array}$ & -5.5 & -10 & -4.5 & -6.5 & -5 & -5.8 \\
\hline Balance water content $\varpi_{e q}(\%)$ & 3 & 4 & 5 & 9 & 4 & 3 \\
\hline
\end{tabular}

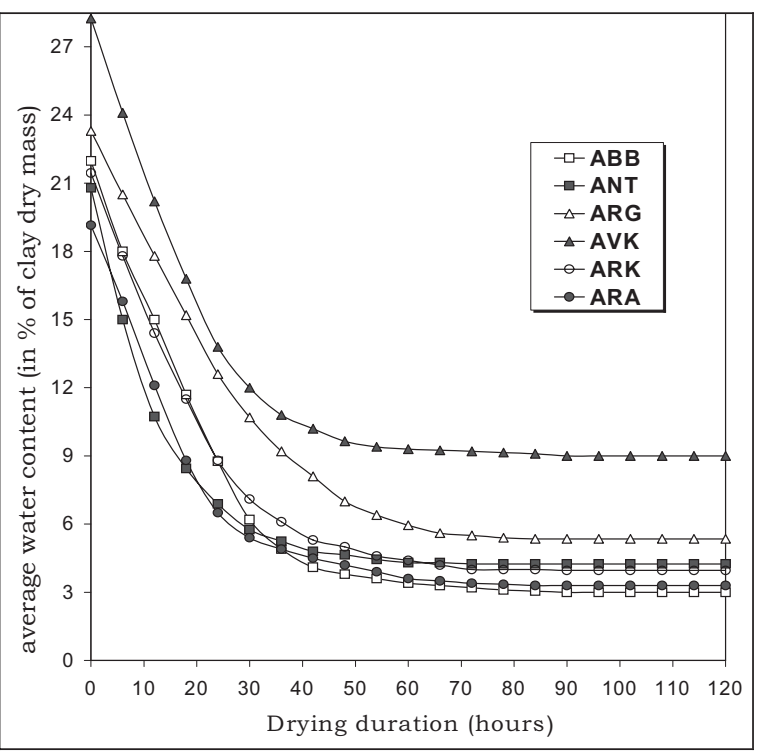

Fig. 4. Variation of the average water content of clay matrixes during drying.

- A second stage in which the drying velocity decreases gradually to zero. When the velocity is about zero, the matrix is in hygroscopic balance with the drying air and its water content does not vary any more. This water content is the balance water content $\varpi_{e q} \cdot \varpi_{e q}$ depends on the hygroscopic characteristics of the drying air and its affinity with a considered clay. Table 1 shows that the AVK clay presents a more important balance water content, so the more affinity with drying air.

The curves of Figure 4 highlight this affinity with ambient air. Although all the clay pastes are moistened to a water content of $18 \%$, the curves indicate the initial water contents $\varpi(0)\left(\right.$ or $\left.\varpi_{i}\right)$ different from $18 \%$ and variable according to clays. The drying curve of the AVK clay whose balance water content is about $9 \%$ gives for example an initial water content of $28 \%$ (Fig. 4). This value corresponds approximately to the sum of $18 \%$ and $9 \%$.

\subsection{Experimental study of the drying Shrinkage: the representative shrinking curves or Bigot's curves}

\subsubsection{Bigot's curves measurement}

The matrix dimensions variation according to the average water content $L(\varpi) / L o$ is called Bigot's curve (Collard [3], Magnan [6], Puffeney [7], Alviset [9],

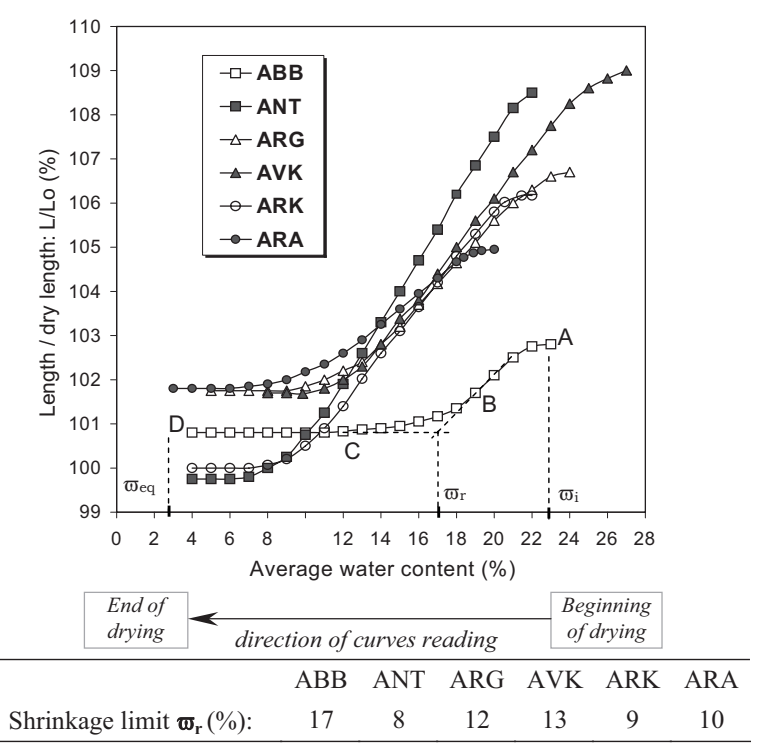

Fig. 5. Representative shrinking curves: Bigot's curves, shrinkage limits.

Sigg [10]). Lo is the length of the dried matrix. This curves expressed the relation between the evaporation and the shrinkage, the shrinkage being due to the moisture evaporation.

\subsubsection{Results and discussions}

Figure 5 gives Bigot's curves of the studied clays and their shrinkage limits.

Bigot's curves allow to determine the shrinking limit $\varpi_{r}$ of these clays. This limit is the value of the water content bellow which the moisture evaporation does no more generate the shrinkage. It corresponds to the abscissa of the intercept point of the linear part of the curve and the tangent of the horizontal part, as illustrated in Figure 5.

The Bigot's curves are interpreted by the shrinkage mechanism occurring in the matrix during drying. This mechanism is illustrated in Figure 6.

Referring to Figures 5 and 6, drying decreases the moisture of the matrix from the shaping water content or initial water content $\varpi_{i}$ to the hygroscopic balance water content $\varpi_{e q}$. The matrix undergoes various transformations:

- from $\varpi_{i}$ to $\varpi_{r}$ (zone AB of Figs. 5 and 6(a)), the matrix dimensions decrease linearly. The moisture evaporation moves solid particles closer together and causes the variation of the matrix dimensions (Fig. 6(a)). The 


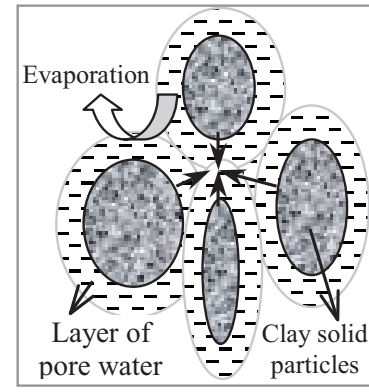

(a) Beginning of drying : evaporation brings particles closer together $\left(\varpi>\varpi_{\mathrm{r}}\right)$

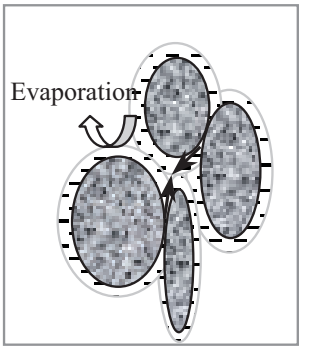

(b) During drying : some particles are in contact ( $\varpi$ is close to $\varpi_{\mathrm{r}}$ )

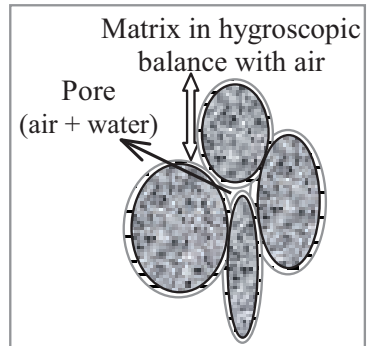

(c) Hygroscopic balance $\left(\varpi=\varpi_{\mathrm{eq}}\right)$

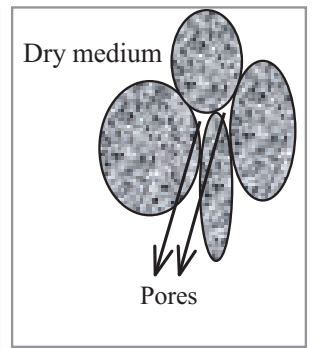

(d) End of drying: all particles are in contact ( $=0)$

Fig. 6. Schematic representation of the shrinking mechanism in a clay matrix during drying.

matrix volume variation is proportional to the volume of the evaporated water: shrinkage is linear;

- around $\varpi_{r}$, (zone BC, Fig. 5 and $6(\mathrm{~b})$ ), some solid particles are already in contact and the variation of matrix dimensions decreases. The slope of the curve decreases as the number of particles in contact increases. The matrix volume variation is no more proportional to the evaporated water volume;

- from $\varpi_{r}$ to $\varpi_{e q}$ (zone CD of Fig. 5 and 6(c), 6(d)), the shrinkage is almost finished. Almost all of the solid particles are in contact and the water evaporation does not any more bring clay particles together. The dimensions of the matrix will remain constant until its complete dehydration (Fig. 6(d)).

\section{Modelling of the moisture transfer in a clay matrix}

During the clay matrix drying, the water evaporation is carried out by a mechanism of transfer due to the water content gradient. In a dissymmetric drying, the water transfer generates internal stresses in the matrix that causes the matrix deformation. The modelling of the water transfer in a clay matrix will make it possible to better understand that mechanism and to optimise clay matrixes drying.

\subsection{Moisture transfer by diffusion: diffusive model for the clay matrix drying}

There are several modes of water transport through a porous structure. There are transfers by diffusion, by capillarity, by pressure gradient, by gravity (Collard [3], Charpin and Rasneur [15]). But according to many authors among whom Sherwood [16], Lewis [17], the transfer by diffusion is the most important mode of water transfer in a porous structure. A diffusive model based on Fick's laws is representative of the water transfer in a clay matrix during drying.

Let $\mathrm{S}\left(x_{1}, x_{2}, x_{3}\right)$ be a point located at the centre of a volume element of the matrix and of which elementary volume is $d x_{1} \cdot d x_{2} . d x_{3}$; let $\omega\left(x_{1}, x_{2}, x_{3}, t\right)$ be the

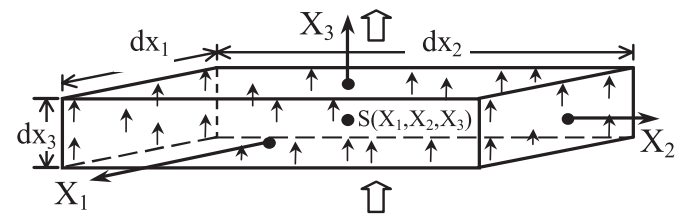

Fig. 7. Water diffusion in a volume element $d x_{1} \cdot d x_{2} \cdot d x_{3}$.

water content at this point at a given time $t$ (Fig. 7). Let us consider the flow of water diffusing along the axis $x_{3}$ (Fig. 7).

The equations of Fick's diffusion laws (Collard [3], Crank [18]) are expressed by:

$$
\begin{aligned}
& \left\{\begin{array}{l}
\Phi_{1}=-\left[D_{11} \frac{\partial \omega}{\partial x_{1}}+D_{12} \frac{\partial \omega}{\partial x_{2}}+D_{13} \frac{\partial \omega}{\partial x_{3}}\right] \\
\Phi_{2}=-\left[D_{21} \frac{\partial \omega}{\partial x_{1}}+D_{22} \frac{\partial \omega}{\partial x_{2}}+D_{23} \frac{\partial \omega}{\partial x_{3}}\right] \\
\Phi_{3}=-\left[D_{31} \frac{\partial \omega}{\partial x_{1}}+D_{32} \frac{\partial \omega}{\partial x_{2}}+D_{33} \frac{\partial \omega}{\partial x_{3}}\right]
\end{array}\right. \\
& \frac{\partial \omega}{\partial t}=\frac{\partial}{\partial x_{1}}\left(D_{1} \frac{\partial \omega}{\partial x_{1}}\right)+\frac{\partial}{\partial x_{2}}\left(D_{2} \frac{\partial \omega}{\partial x_{2}}\right)+\frac{\partial}{\partial x_{3}}\left(D_{3} \frac{\partial \omega}{\partial x_{3}}\right) .
\end{aligned}
$$

$\Phi_{i}$ is the flux density according to the direction $x_{i}, D_{i j}$ the diffusion coefficient $\left(\mathrm{m}^{2} / \mathrm{s}\right), \omega\left(x_{1}, x_{2}, x_{3}, t\right)$ the water content at a point $\mathrm{S}\left(x_{1} ; x_{2} ; x_{3}\right)$ of the matrix at a time $t$.

In an isotropic medium where the coefficients $D_{i j}$ are identical in every direction, and by considering them constant, and in the case of a flat shape matrix where the flux of water diffusion is unidirectional and carried out according to the axis $x_{3}$, the above equations are simplified (Crank [18], William and Callister [19], Shelby [20], Asheland [21], Evans and Keey [22]):

$$
\Phi=-D \frac{\partial \omega}{\partial x_{3}} \quad \text { and } \quad \frac{\partial \omega}{\partial t}=D \frac{\partial^{2} \omega}{\partial x_{3}^{2}} .
$$

The solutions of these equations depend on the initial conditions and the boundary conditions, as showed by Crank [18]. These conditions are illustrated in Figure 8. 


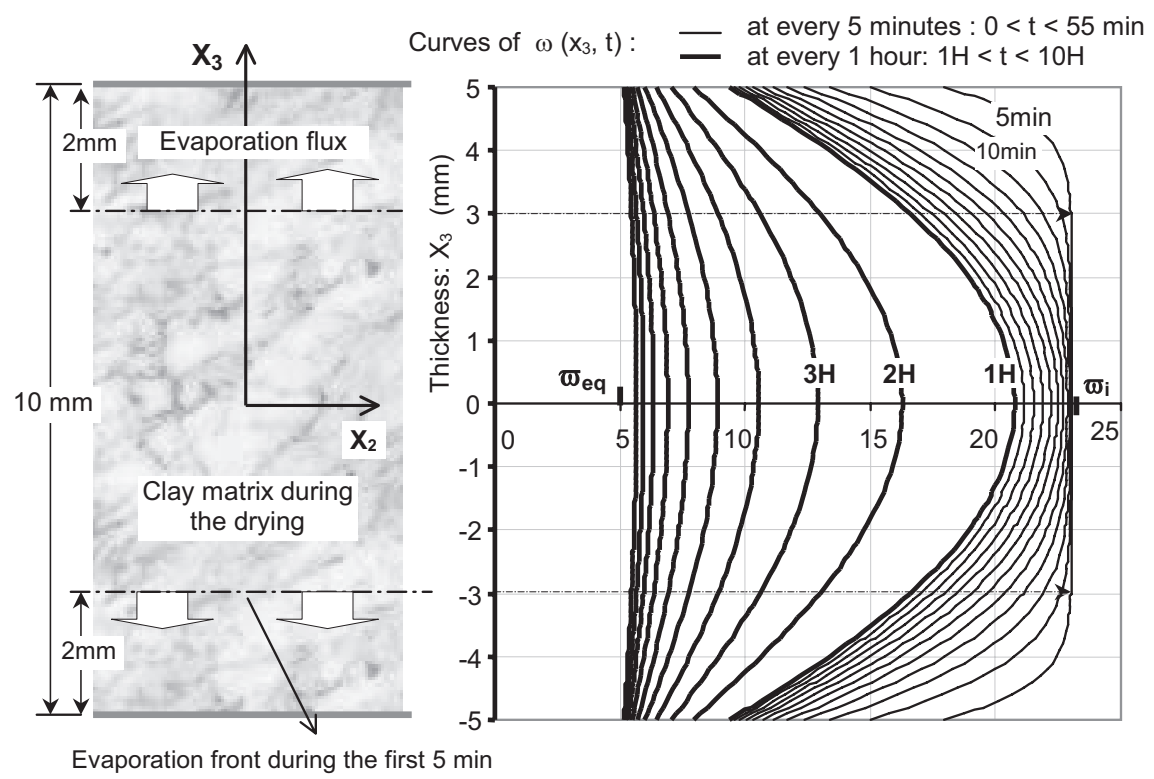

Fig. 9. Simulation of a flat clay matrix drying (case of the red clay of Guérin-Kouka): evolution of the water content at every $5 \mathrm{~min}$ and at every $1 \mathrm{~h}$.

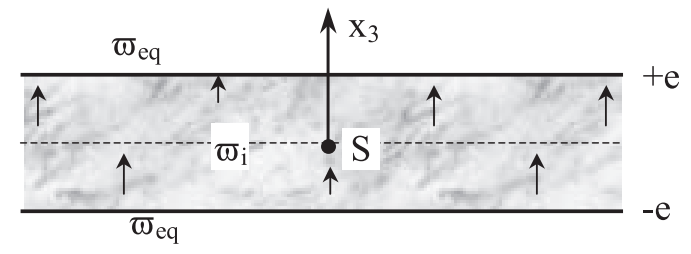

Fig. 8. Initial and boundary conditions.

The initial conditions and the boundary conditions are then expressed as follows:

$$
\begin{aligned}
& \omega\left(x_{3}, t\right)=\varpi_{i} \text { for }-e<x_{3}<+e \text { and } t \leqslant 0 \\
& \omega\left(x_{3}, t\right)=\varpi_{e q} \text { for } x_{3}= \pm e \text { and } t>0 .
\end{aligned}
$$

The solution giving the value of the water content at each point of the clay flat matrix at every time during drying is expressed as follows (Collard [3], PolubarinovaKochina [12], Crank [18]):

$$
\begin{aligned}
& \frac{\omega\left(x_{3}, t\right)-\varpi_{e q}}{\varpi_{i}-\varpi_{e q}}= \frac{4}{\pi} \sum_{k=0}^{\infty} \frac{(-1)^{k}}{(2 k+1)} \operatorname{Cos}\left(\frac{(2 k+1) \cdot \pi}{2 e} x_{3}\right) \\
& \times \operatorname{Exp}\left(-\frac{(2 k+1)^{2} \pi^{2}}{4 e^{2}} D t\right) \\
& \forall x_{3} \in[-e,+e] \quad \text { and } \quad \forall t \geqslant 0 .
\end{aligned}
$$

From the above expression of $\omega\left(x_{3}, t\right)$, the average water content $\varpi(\mathrm{t})$ of the whole matrix can be deducted as:

$$
\varpi(t)=\frac{1}{2 e} \int_{-e}^{e} \omega\left(x_{3}, t\right) d x_{3} \text { or }
$$

$$
\overline{{\frac{\varpi(t)-\varpi_{e q}}{\varpi}}_{i}-\varpi_{e q}=\frac{8}{\pi^{2}} \sum_{k=0}^{\infty} \frac{1}{(2 k+1)^{2}} \operatorname{Exp}\left(-\frac{(2 k+1)^{2} \pi^{2}}{4 e^{2}} D t\right)}
$$$$
\forall t \geqslant 0 \text {. }
$$

\subsection{Modelling results and discussion}

The diffusion coefficient has been determined experimentally and its value during a transitory water diffusion regime differs slightly from that of a permanent regime:

- during the transitory regime: $D=10^{-9} \mathrm{~m}^{2} / \mathrm{s}$;

- during the permanent regime: $D=10^{-10} \mathrm{~m}^{2} / \mathrm{s}$.

These values are very closed to those given by the literature (Collard [3], Evans and Keey [22]). The simulation is carried out on a flat matrix of $10 \mathrm{~mm}$ of thickness.

The initial water content $\varpi_{i}$ and the balance water content $\varpi_{e q}$ are given by Table 1 and Figure 4 .

In the case of the red clay of Guérin-Kouka (ARG): $\varpi_{i}=23 \% ; \varpi_{e q}=5 \%$ and $e=5 \mathrm{~mm}$.

The computer calculation is reduced to the first 52 terms of the sum of the above equations or $0 \leqslant k \leqslant 51$.

Figure 9 gives the evolution of the water content at every 5 min during the first 55 min of drying and at every $1 \mathrm{~h}$ during the first $10 \mathrm{~h}$.

Figure 9 shows that the evaporation front progresses from faces towards the middle of the matrix thickness. At the end of the first $5 \mathrm{~min}$, the evaporation front is at $2 \mathrm{~mm}$ from faces, the matrix interior still having the initial water content $\varpi_{i}$. Evaporation front reaches the middle of the matrix thickness after a half an hour of drying. The water exchange balance will be established only after 30 to $40 \mathrm{~h}$ of drying.

At this point the balance water content $\varpi_{e q}$ is about $5 \%$.

The unequal water content $\omega\left(x_{3}, t\right)$ (Fig. 9) in the matrix during drying presents two consequences:

- evaporation generates the matrix shrinkage that is increasingly important from faces towards the matrix thickness middle. This generates internal tensions and stresses correlatively more important from faces 


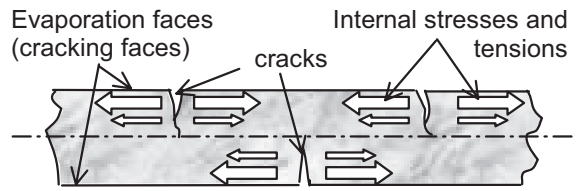

(a) Symmetrical evaporation: internal tensions and stresses are more important from faces towards the middle of the flat matrix thick: appearance of cracks on the faces

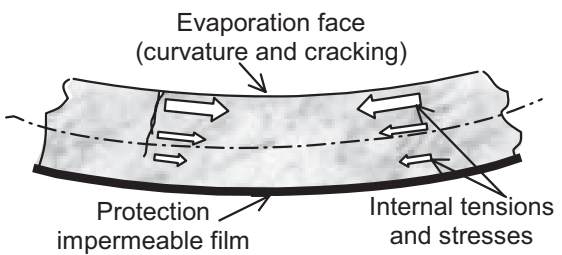

(b) Dissymmetrical evaporation: dissymmetrical distribution of internal stresses in a flat matrix of which a face is covered with an impermeable film: curvature on the side of the evaporation face

Fig. 10. Internal stresses distribution in a flat matrix thickness during a symmetrical drying (a) and a dissymmetrical drying (b).

towards the matrix thickness middle (Puffeney [7], Jouenne [13], Bottin [14]). When these internal stresses and tensions are important, they generate the matrix cracking, the cracks occurring on the flat faces as illustrated in Figure 10(a) (Collard [3], Jacquemin [23], Paul [24]). To reduce the internal stresses and tensions, the evaporation velocity must be controlled and lowered;

- when the two faces of a flat matrix are not exposed in an identical manner to the drying air, the dissymmetrical resulting evaporation generates a dissymmetrical shrinkage and correlatively an unequal distribution of tensions and stresses on the two faces. This causes the curvature of the flat. The concavity appears on the face which is most exposed to the air because of its more important shrinkage and its more important internal stresses and tensions.

This is observed when drying is performed on a clay flat matrix of which one of its faces is covered with an impermeable film in order to reduce the evaporation on that face (Fig. 10(b)) (Collard [3], Alviset [9]).

The simulation results can be validate by superimposing the experimental curve of the average water content and the calculated average water content $\varpi(\mathrm{t})$ (Fig. 11).

The calculated $\varpi(t)$ is reduced to the first 52 terms of the sum of the equation of $\varpi(t)$ :

$$
\varpi(t)=5+\frac{144}{\pi^{2}} \sum_{k=0}^{50} \frac{1}{(2 k+1)^{2}} \operatorname{Exp}\left(-10^{-6} \pi^{2}(2 k+1)^{2} t\right)
$$$$
\forall t \geqslant 0 \text {. }
$$

Referring to Figure 11, the maximum error resulting from the simulation is about $10 \%$. In spite of this high value of the error, the experimental curve and the simulated curve are globally close and superimposed. This coincidence allows to validate the Fick's diffusive model applied to the clay matrixes drying.

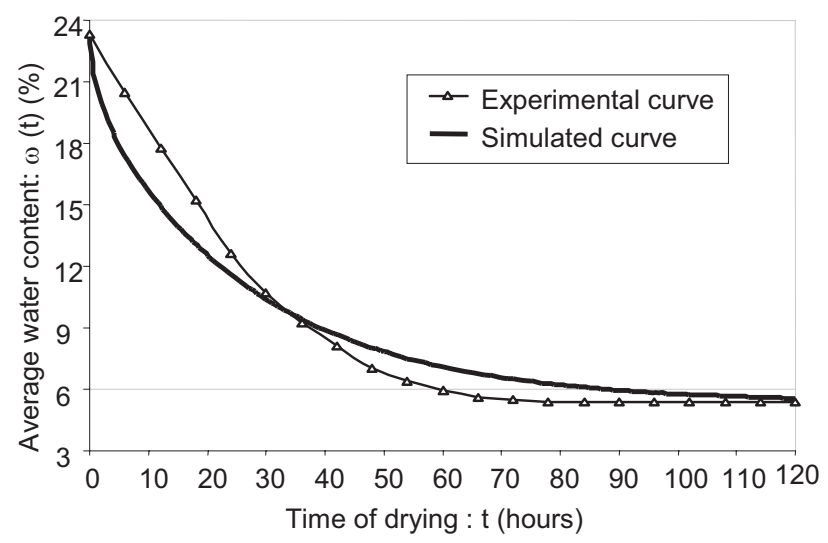

Fig. 11. Superimposition of the experimental curve and the simulated curve giving the average water content in the clay matrix during drying.

\section{Conclusion}

The validation of the simulation model allows to apply the experimental results and the modelling results to the drying of the various studied clay varieties.

A clay matrix drying comprises a critical zone corresponding to a zone where the water content of the matrix is still above its shrinkage limit and a zone without risks corresponding to a zone where the water content is below the shrinkage limit.

During the critical zone, the risks of the matrix cracking and deformation are more important when the evaporation velocity is important and when the evaporation is dissymmetrical.

To avoid cracking and deformations, drying must be controlled and performed with precautions. These precautions are summarized in Table 2. Table 2 gives some advised evaporation velocities required according to the clay variety. The evaporation must not exceed these velocity values in order to prevent matrixes from cracking 
Table 2. Drying parameters and controlling advisable for the six clay varieties matrixes drying.

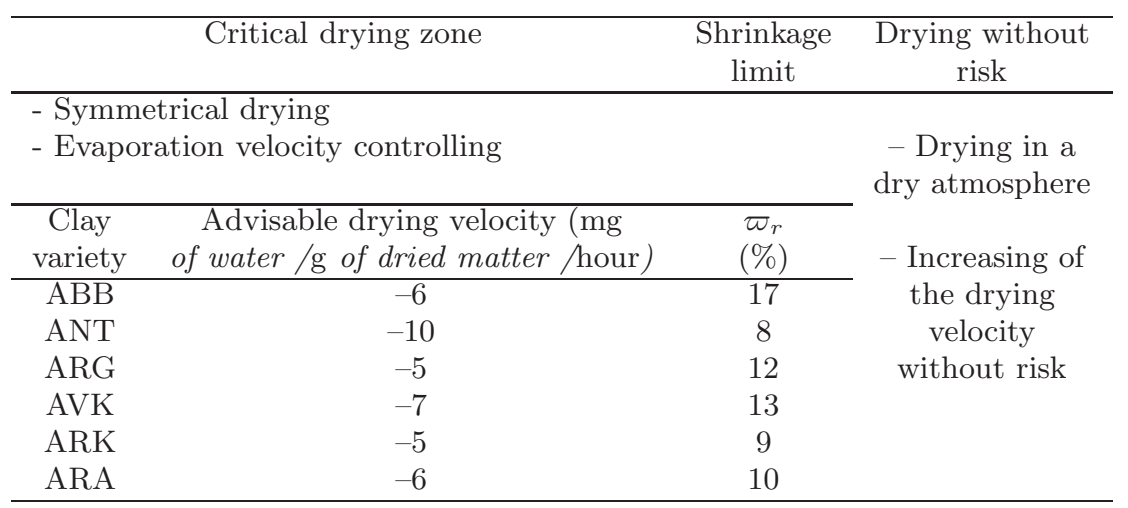

and deforming. For this purpose, drying will be performed in a wet atmosphere or in a quasi hermetic medium for example (see Tab. 2).

During the zone without risk o below the shrinkage limit, drying can be continued in a dry atmosphere and the evaporation velocity can be increased without risk.

\section{References}

1. K.-E. Atcholi, Rapport d'activités de Recherches, Université de Lomé, Février 1997

2. E., Padayodi Contribution à l'élaboration des matériaux de construction à base de produits naturels : caractérisation thermomécanique et physico-chimique des argiles et des fibres naturelles cellulosiques, Thèse UFR Sciences et Techniques, Université de Franche-Comté (2001)

3. J.-M. Collard, Étude des transferts d'humidité et des déformations pendant le séchage d'une plaque d'argile, Thèse de l'Université de Poitiers (1989)

4. R. Ho-Yick-Cheong, La brique de terre crue comprimée et stabilisée au ciment: caractéristiques et propriétés physico-mécaniques, Thèse de l'Université Joseph-FourierGrenoble I (1989)

5. CDI Centre pour le Développement Industriel (A.C.PC.E.E), Blocs de terre comprimée: choix du matériel de production, 1 ère édition, Sept. 1988

6. J.-P. Magnan, Description, identification et classification des sols, Technique de l'Ingénieur, Vol. C2I, N C208. Ed. Istral BL (1996)

7. J.-P. Puffeney, Système à base de connaissances d'aide à la décision pour la conduite d'une tuilerie automatisée, Thèse UFR Sciences et Techniques, Université de FrancheComté (1997)

8. C. Suri, Étude du couplage des phénomènes d'absorption et d'endommagement dans un composite verre-epoxy, Thèse UFR Sciences et Tech., Univer. de Franche-Comté (1995)
9. L. Alviset, Matériaux de terre cuite, Technique de l'Ingénieur, Vol. C1, N C905. Ed. Istral BL (1996)

10. J. Sigg, Les produits de terre cuite, Paris, Septima (1991)

11. Publication du Centre Technique des Tuiles et Briques, Facteurs influençant le séchage, 30 pages

12. P.Ya Polubarinova-Kochina, Theory of ground water movement (Gostekhizdat, Moscou), Princeton Univ. Press, N.J. (1962)

13. C.A. Jouenne, Traité de Céramiques et Matériaux Minéraux, Editions Septima. Paris. 1980, pp. 368-500

14. C. Bottin, La fabrication artisanale de tuiles romanes, Edition CRATerre (1988)

15. Charpin, Rasneur, Mesure des surfaces spécifiques, Technique de l'Ingénieur, Analyse et caractérisation, $\mathrm{N}^{\circ} \mathrm{P} 1050$ (1987).

16. T.K. Sherwood, The drying of solid, Tome I and II $\mathbf{2 1}$, 12-16 and 976-980 (1929)

17. R.W. Lewis, Drying induced stresses in porous bodies. Int. J. Numer. Meth. Eng. 11, 1175-1184 (1977)

18. J. Crank, The Mathematics of diffusion, Clarendon Press, Oxford (1975)

19. D. William, Jr. Callister, Materials Science and Engineering, Dep. of Metal. Eng. of The University of Utah. 4th edn., 90-100 (1996)

20. J.A. Shelby, Introduction to glass science and technology, New York State College of Ceramics and Alfred Univ., 158-160 (1997)

21. Donald R. Askeland, The Science and Engineering of Materials, 3th ed., 429-430 (1994)

22. A.A. Evans, R.B. Keey, The water diffusion coefficient of an shrinking clay on drying. Chem. Eng. J., 126-135 (1975)

23. F. Jacquemin, Modélisation et simulation des contraintes internes dans les structures tubulaires composites épaisses, Thèse de l'Université Blaise Pascal-Clermont II (2000)

24. D. Paul, Contraintes Hygrothermiques dans les matériaux composites stratifiés : Modélisation et mise en évidence expérimentale, Thèse de l'université Jean Monnet de Saint-Etienne (1996) 